\title{
Clinical significance of stromal ER and PR expression in periampullary adenocarcinoma
}

Gustav Andersson *iD, Sebastian Lundgren, Margareta Heby, Björn Nodin, Jacob Elebro and Karin Jirström

\begin{abstract}
Background: Tamoxifen treatment has previously been reported to confer life-prolonging effects in patients with advanced pancreatic cancer, and most evidently so in women. None of these trials did however include biomarkers, and the relevance of female hormone signaling in pancreatic or other periampullary adenocarcinoma remains largely unexplored. The aim of this study was to examine the extent and potential clinical significance of estrogen receptor-a (ER) and progesterone receptor (PR) expression in pancreatic and other periampullary cancers.

Methods: ER and PR expression was examined using immunohistochemistry on tissue microarrays with primary tumors from a retrospective consecutive cohort of 175 patients with resected periampullary adenocarcinoma, with long-term clinical follow-up. Non-parametric and Chi square tests were applied to examine the associations of stromal ER and PR expression with patient and tumor characteristics. Kaplan-Meier analysis and log rank test were applied to illustrate survival differences in relation to ER and PR expression. Cox regression proportional hazards models were applied to examine the associations between investigative factors and risk of death and recurrence, and to test for interactions between KRAS mutation status and hormone receptor expression in relation to survival.
\end{abstract}

Results: Expression of both ER and PR was more frequent in the tumor-associated stroma than in the epithelium. A significant prognostic interaction, independent of tumor morphology, was found between stromal PR expression and KRAS mutation status in relation to both overall and recurrence-free survival ( $p_{\text {interaction }}=0.026$ and $p_{\text {interaction }}=$ 0.005), in particular in women ( $p_{\text {interaction }}=0.002$ and $P_{\text {interaction }}=0.005$ ). Specifically, stromal PR expression was associated with a prolonged survival in patients with KRAS-mutated tumors, whereas the opposite was seen for KRAS wild-type tumors. The prognostic value of ER positivity was limited to the subgroup of women with tumors of pancreatic origin.

Conclusions: These results demonstrate that stromal PR rather than ER expression, together with KRAS mutation status, provides long-term prognostic information in patients with periampullary adenocarcinoma. Further study into the mechanistic basis for these observations may unveil important clues to the pathogenesis of these cancers and open up for the discovery of novel treatment options.

Keywords: Periampullary adenocarcinoma, Pancreatic cancer, Estrogen receptor, Progesterone receptor, KRAS, Prognosis

\footnotetext{
* Correspondence: gustav.andersson@med.lu.se

Division of Oncology and Pathology, Department of Clinical Sciences Lund,

Lund University, Lund, Sweden
}

(c) The Author(s). 2019 Open Access This article is distributed under the terms of the Creative Commons Attribution 4.0 International License (http://creativecommons.org/licenses/by/4.0/), which permits unrestricted use, distribution, and reproduction in any medium, provided you give appropriate credit to the original author(s) and the source, provide a link to the Creative Commons license, and indicate if changes were made. The Creative Commons Public Domain Dedication waiver (http://creativecommons.org/publicdomain/zero/1.0/) applies to the data made available in this article, unless otherwise stated. 


\section{Background}

Pancreatic cancer is an almost uniformly fatal disease, from which most patients decease within 1 year, and thus, the incidence approximates the prevalence [1]. It is the most common tumor within the clinical entity of periampullary tumors, including tumors originating in the distal bile duct, pancreas, ampulla of Vater and the periampullary duodenum. Information on morphological type, i.e. intestinal (I-type) or pancreatobiliary type (PBtype), has been shown to provide prognostic information, with the latter having the poorest outcome [2-5]. Only $15-20 \%$ of the tumors are resectable at presentation [6]. Of the remaining majority, 30-40\% present with locally advanced tumor growth, leaving almost 40-55\% of all patients with stage IV disease [7]. Recent and growing data support the use of neoadjuvant chemotherapy, and for patients presenting with a borderline resectable tumor, approximately one-third can be converted to resectability resulting in a doubled overall survival compared to those who remain unresectable [8]. Adjuvant chemotherapy is the standard of care following resection for pancreatic adenocarcinoma. In the palliative setting, survival is strictly limited, and only modestly improved by chemotherapy $[9,10]$. Although the vast majority of pancreatic cancers contain somatic mutations [11], there is still a complete lack of "actionable" molecular targets, and immunotherapies have not proven to be successful [12]. Accordingly, there is an urgent need for novel treatment alternatives and suitable biomarkers to improve the outlook for these patients. In this context, it is noteworthy that several trials in the 1980 s to 1990 s reported beneficial effects of tamoxifen treatment in patients with pancreatic adenocarcinoma [13-15], primarily in women with advanced disease [16, 17]. None of these studies did however include biomarker analyses, nor did they consider the full range of periampullary cancers.

Whilst the role of female hormone signaling in established pancreatic adenocarcinomas remains unclear, several studies, including research from our group, have suggested an inverse relationship between exogenous and endogenous female sex hormones and risk of pancreatic cancer [18-26]. Contrasting findings have however been presented by others [23, 25, 27-31].

Evidently, the interplay between hormonal factors and pancreatic cancer is complex and far from being fully understood. Only a few previous studies have examined the expression of estrogen receptor- $\alpha$ (ER) and progesterone receptor (PR) in pancreatic or other periampullary adenocarcinomas [32, 33], and only the latter assessed their prognostic value. The aim of the present study was therefore to investigate the expression of ER and PR in tumors from a well-characterized, consecutive retrospective cohort of resected periampullary adenocarcinoma, with particular emphasis on their relationship with the mutational landscape, clinicopathological characteristics and long-term survival.

\section{Methods \\ Study cohort}

The study cohort, previously described in more detail [34-38], is a retrospective consecutive cohort encompassing 175 patients diagnosed with pancreatic or other periampullary adenocarcinomas, 65 cases with I-type morphology and 110 cases with PB-type morphology. All tumors were surgically resected through the Whipple procedure (pancreaticoduodenectomy) between Jan 1st 2001 and Dec 31st 2011, in Malmö and Lund University Hospitals, Sweden. Hematoxylin and eosin stained tumor slides from all cases were re-evaluated by a board-certified pathologist (JEL), blinded to original reports and patient outcomes. Evaluation of tumor origin and morphology was based on previously described criteria [34].

Baseline was set at the date of surgery, the primary endpoint was overall survival (OS) and the secondary endpoint recurrence-free survival (RFS), set at date of death and date of recurrence respectively, or March 31st 2017, the last date of follow-up, whichever came first. The Swedish National Civil Register provided data on survival, and patient records were reviewed for information on neoadjuvant and adjuvant treatment, and tumor recurrence. At last follow-up, 137 patients were dead, and 124 had denoted recurrence. The median follow-up time for the entire cohort was 29.7 (range 1.9-185.1) months, and 86.1 (range 63.5185.1) months for patients alive [39].

\section{Tissue microarray construction}

Tissue microarrays (TMAs) were constructed using a semi-automated arraying device (TMArrayer, Pathology Devices, Westminster, MD, USA). A standard set of three tissue cores ( $1 \mathrm{~mm}$ diameter) were obtained from each of the 175 formalin-fixed paraffin-embedded (FFPE) primary tumors, whenever possible from different donor blocks, and one to three tissue cores from lymph node metastases of 105 cases. Additionally, nonmalignant pancreatic tissue samples were obtained from 50 of the resection specimens, using a standard set of two tissue cores ( $1 \mathrm{~mm}$ diameter).

\section{Immunohistochemistry and staining evaluation}

For immunohistochemical analysis, $4 \mu \mathrm{m}$ TMAsections were automatically pre-treated using ULTRA Cell Condition Solution 1, pH 8.5 (Ventana Medical Systems, Tucson, AZ, USA) for heat induced epitope retrieval, and stained with the monoclonal antibodies CONFIRM anti-ER (SP1) and anti-PR (1E2) in a 
Ventana BenchMark stainer (Ventana Medical Systems). The antibody-antigen complex was visualized with UltraView Universal diaminobenzidine (DAB) Detection kit (Ventana Medical Systems).

Stained slides were digitalized at 20x magnification using the automated scanning system Hamamatsu, NanoZoomer, 13239-01, Hamamatsu Photonics, Sunayama-cho, Naka-ku, Hamamatsu City, Shizuoka, Japan). The immunohistochemical staining was manually annotated by three independent observers (GA, KJ and JEL) who were blinded to the clinical data and outcome of the patients, and with the two latter being board-certified pathologists, using the NDP.view2 viewer software version 3.2.12 (Hamamatsu Photonics). Expression of ER and PR was annotated as the number of tumor-associated stromal cells with nuclear staining. Conflicting annotations were jointly re-evaluated in order to reach consensus. The intensity of staining was not evaluated.

\section{Next generation sequencing}

DNA extraction was performed on tissue cores $(1 \mathrm{~mm}$ diameter), obtained alongside the TMA cores from tumor cell-enriched FFPE tissue, with the Qiagen GeneRead (Qiagen, Hilden, Germany) kit for FFPE tissue in accordance with instructions from the manufacturer. For analysis, a sufficient quantity of tumor cells could be obtained from $102(58.3 \%)$ cases, 40 (39.2\%) I-type tumors and 62 (60.8\%) PB-type tumors, including 10 duodenal, 42 ampullary, 30 distal bile duct and 20 pancreatic tumors. A gene panel with selected cancer-associated genes $(n=70)$ (see Additional file 1: Data 1$)$ was assembled and characterized using Illumina TruSeq custom amplicon assay (Illumina Inc., San Diego, USA) with a MiSeq instrument according to the manufacturer's instructions, previously described in more detail by Lundgren et al. [40]. Only exon parts of the analyzed genes were sequenced. The supplier's standard analysis pipeline (Illumina Inc., San Diego, CA, USA) was applied for alignment, quality filtering, variant calling, and variant annotation. Only nonsynonymous variants with variant frequency $\geq 4 \%$ were included. In order to exclude SNPs frequently reported in various populations, the identified mutations were screened against the COSMIC and ExAC databases.

\section{Statistical analysis}

In the statistical analyses, ER and PR expression were defined as continuous variables as well as binary variables, dichotomized into positive and negative, i.e. presence vs absence of positive stromal cells. Spearman's rank correlation test was used to examine the intercorrelation between the numbers of ER positive $(E R+)$ and $\mathrm{PR}$ positive $(\mathrm{PR}+)$ stromal cells allover, and stratified for sex and morphology. Non-parametric and Chi square tests were applied for analysis of the associations of stromal ER and PR expression with patient and tumor characteristics in the entire cohort, as well as in strata according to sex and morphology. The distribution of mutations in relation ER and PR expression, stratified by sex and morphology, was visualized by heat maps created in OncoPrinter, provided by cBioPortal Web (http://www.cbioportal.org/oncoprinter.jsp, access date: 2018-11-05) [41, 42]. Kaplan-Meier analysis and log rank test were applied to illustrate differences in OS and RFS stratified by sex, morphology, and KRAS mutation status in relation to ER and PR expression. Univariable and multivariable Cox regression proportional hazards modeling was applied to estimate hazard ratios (HR) and 95\% confidence intervals (CI) for risk of death and recurrence. The multivariable analysis included adjustment for age at baseline (continuous), tumor morphology (Itype/PB-type), T-stage (pT1-2/pT3-4), N-stage (pN0/ $\mathrm{pN} 1$ ), tumor grade (well-moderate differentiation/poor differentiation-undifferentiated), adjuvant chemotherapy treatment (no/yes), lymph vessel invasion (no/yes), blood vessel invasion (no/yes) and perineural invasion (no/yes). Cox regression proportional hazards modeling was also used to test for potential interactions between KRAS mutation status and biomarker (ER/PR) expression in relation to survival, using the following interaction variable: KRAS mutation $(0 / 1) \mathrm{x}$ biomarker $(0 / 1)$.

All statistical tests were two sided and $p$-values $<0.05$ were considered significant. As this study is considered exploratory, the presented nominal $p$-values were not adjusted for multiple testing [43]. All statistical analyses were computed using IBM SPSS Statistics version 25.0 (SPSS Inc., Chicago, IL, USA).

\section{Results}

\section{Distribution of ER and PR expression, overall and stratified for sex and morphology}

ER and PR expression was mainly detected in the peritumoral stroma. Weak epithelial expression of ER and PR was denoted in $2.9 \%$ of the cases and therefore not accounted for in the statistical analyses. Neuroendocrine islets frequently displayed moderate to strong nuclear expression of ER and PR.

The distribution of ER and PR expression among the five different tumor origins is shown in Fig. 1, along with sample immunohistochemical images of stromal ER and PR expressing cells. Stromal ER positivity, allover denoted in $29.0 \%$ of the cases, with numbers ranging from 2 to 100 positive cells, was equally distributed between I-type and PB-type tumors $(31.1 \%$ vs $25.4 \%, p=$ $0.43)$, but was more common in women compared to men (entire cohort: $41.7 \%$ vs $16.5 \%, p<0.001$ and PBtype tumors: $46.0 \%$ vs $17.9 \%, p=0.002$ ). However, the 
A

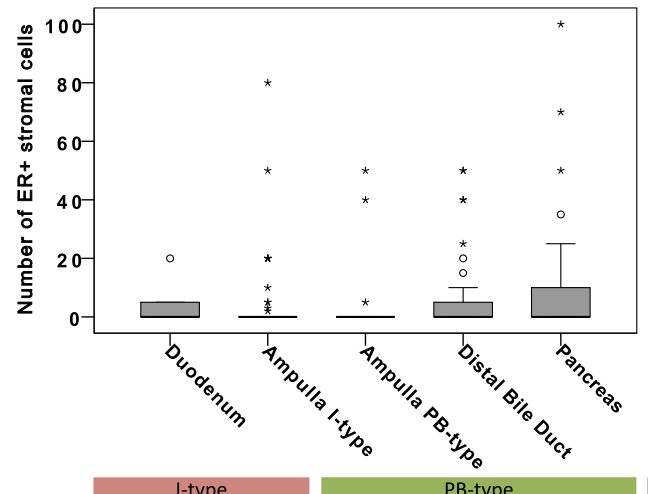

C

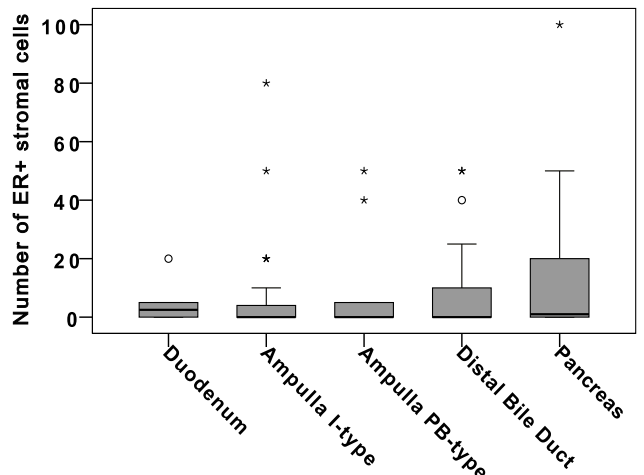

l-type PB-type P=0.258

\section{E}

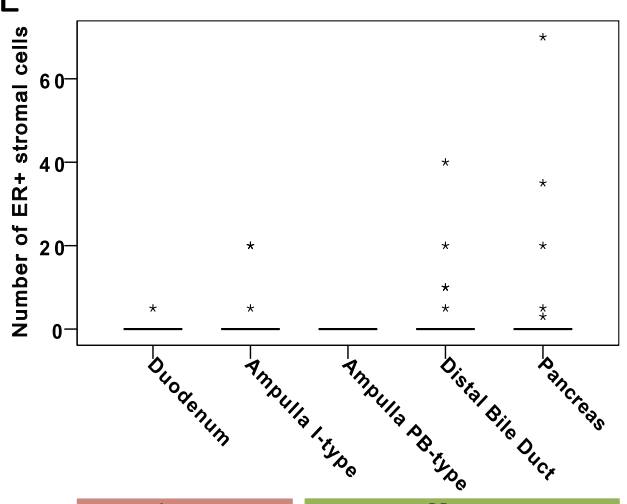

B

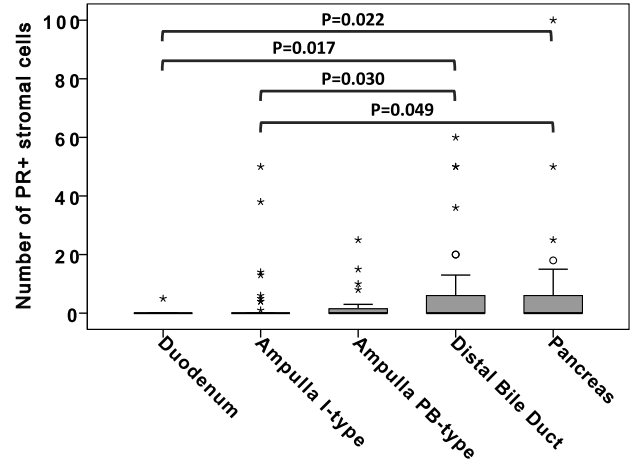

I-type

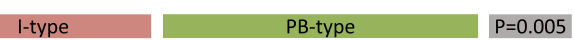

D
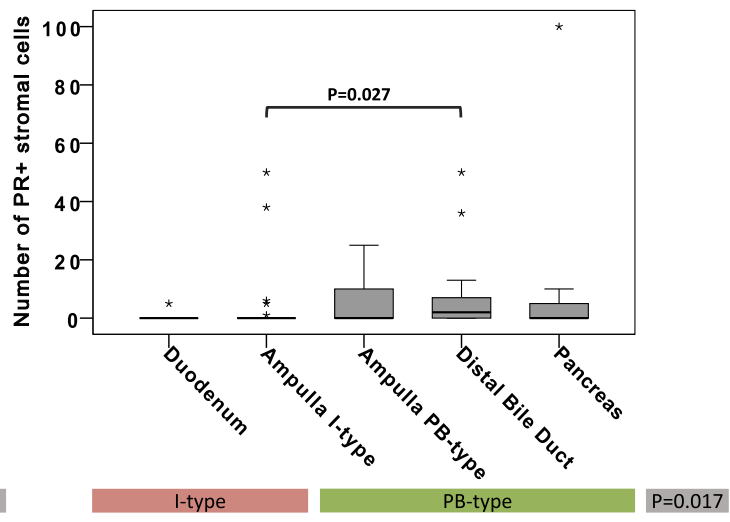

$\mathrm{F}$

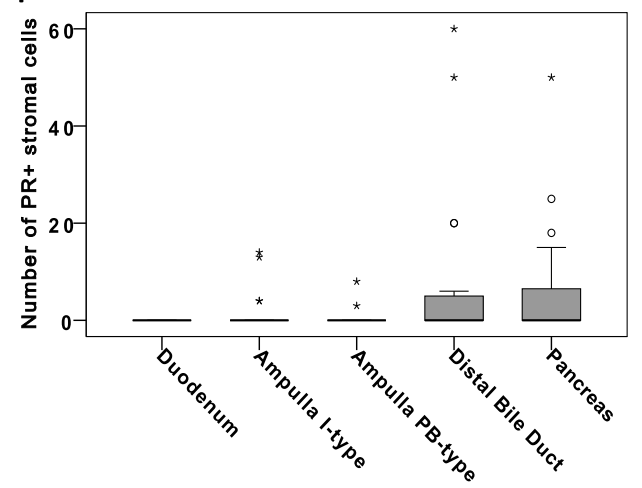

$P=0.595$

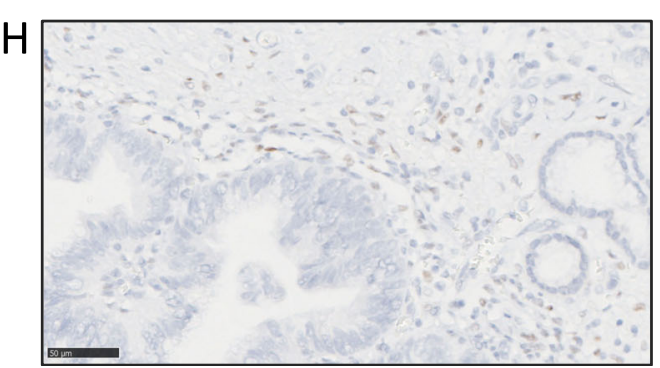

Fig. 1 (See legend on next page.) 
(See figure on previous page.)

Fig. 1 Distribution of hormone receptor positive stromal cells in relation to tumor origin and morphology. Box plots illustrating the distribution of stromal cells expressing ER ( $(\mathbf{a}, \mathbf{c}, \mathbf{e})$ and PR $(\mathbf{b}, \mathbf{d}, \mathbf{f})$ among the five different tumor origins, in the entire cohort $(\mathbf{a}-\mathbf{b})$, in women $(\mathbf{c}-\mathbf{d})$ and in men (e-f). P-values were measured with non-parametric test. Sample immunohistochemical images of stromal ER and PR expression, respectively, are shown in panels $(\mathbf{g}$ and $\mathbf{h})$

number of ER+ stromal cells was not associated with morphology, neither in the entire cohort nor in subgroup analysis according to sex. Stromal PR positivity, allover observed in $30.7 \%$ of the cases, with numbers ranging from 1 to 100 positive cells, was significantly more common in PB-type than in I-type tumors overall ( $38.1 \%$ vs $17.2 \%$ positivity, $p=0.006)$, and in women ( $45.8 \%$ vs $18.8 \%$ positivity, $p=0.013$ ). Similarly, the number of PR+ stromal cells was significantly higher in PBtype than in I-type tumors overall $(p=0.005)$, and in women $(p=0.017)$.

As shown in Additional file 2: Table S1, there was a significant intercorrelation between stromal ER+ and $\mathrm{PR}+$ in the entire cohort $(p<0.001)$, and in subgroup analysis according to sex (women: $p=0.011$, men: $p<0.001)$. In subgroup analysis according to tumor morphology, there was a significant intercorrelation between $\mathrm{ER}+$ and $\mathrm{PR}+$ in PB-type tumors in the entire cohort $(p<0.001)$ and in subgroup analysis according to sex (women: $p=0.036$, men: $p<0.001$ ). There was no significant intercorrelation between $\mathrm{ER}+$ and PR+ in I-type tumors.

\section{Associations of ER and PR with patient and tumor characteristics}

Associations between binary variables of positive and negative ER and PR expression with clinicopathological characteristics are shown in Additional file 3: Table S2 and Additional file 4: Table S3, respectively. All significant associations of the number of ER and PR positive stromal cells with patient and tumor characteristics in the entire cohort, and stratified for morphology and sex, respectively, are shown in Fig. 2. There was a significant positive association between the number of $\mathrm{ER}+$ stromal cells and female sex in the entire cohort and in PB-type tumors. Significant positive associations were also seen between the number of $\mathrm{PR}+$ stromal cells and perineural growth in the entire cohort, lymph vessel invasion in PB-type tumors, blood vessel invasion in men, and $\mathrm{N}$-stage in women.

\section{Distribution of common mutations in relation to ER and PR expression}

Heatmaps of the distribution and type of the nine most common mutations (mutation frequency $>10 \%$ ) stratified by ER and PR status and sex, in the entire cohort and further stratified by morphology, are shown in Figs. 3 and 4 , respectively. The associations between binary tumor mutation status and ER and PR expression, respectively, allover and stratified for sex and morphology, are shown in Additional file 5: Table S4 and Additional file 6: Table S5. ER positivity was significantly associated with SMAD4 mutations in women $(p=0.024)$, and with $A P C$ mutations, exclusively found in I-type tumors [40] $(p=0.032)$. PR positivity was significantly associated with TP53 mutations in men $(p=0.044)$, with SMAD4 mutations in I-type tumors ( $p=0.005$ for all, $p=0.043$ for women), and with ERBB3 wild-type tumors in the entire cohort $(p=0.021)$.

\section{Associations of stromal ER and PR expression with overall and recurrence-free survival}

As shown in Table 1, neither ER nor PR expression in the tumor stroma was prognostic per se in the entire cohort, nor in subgroup analysis according to sex. There was however a significant interaction between PR, but not ER, expression and KRAS mutation status in relation to $\mathrm{OS}$ in the entire cohort ( $\mathrm{p}$ for interaction $=0.026$ ) and in women ( $\mathrm{p}$ for interaction $=0.002$ ).

Similar findings were seen for PR expression and KRAS mutation status in relation to RFS in the entire cohort ( $\mathrm{p}$ for interaction $=0.005)$ and in women $(\mathrm{p}$ for interaction $=0.005$ ), as demonstrated in Table 2. Of note, there was also a significant interaction between ER expression and KRAS mutation status in relation to RFS in men ( $\mathrm{p}$ for interaction $=0.025$ ). However, the impact of ER expression on RFS did not reach significance in any strata according to KRAS mutation status.

As further shown in Tables 1 and 2, the interactions between PR expression and KRAS status in relation to both OS and RFS remained significant after adjustment for morphological type.

The independent prognostic impact of ER and PR expression, respectively, in the entire cohort was also tested in a multivariable model including other established prognostic factors, whereby no significant associations were found (data not shown). Due to the small number of cases, adjustment for other factors than morphological type was not performed in further substrata.

Subgroup analysis according to morphological type did not reveal any significant associations between ER or PR expression with neither OS nor RFS (data not shown). However, subgroup analysis according to anatomical tumor origin revealed that ER positivity was significantly associated with a prolonged OS and RFS in women with tumors of pancreatic origin in univariable analysis (HR 


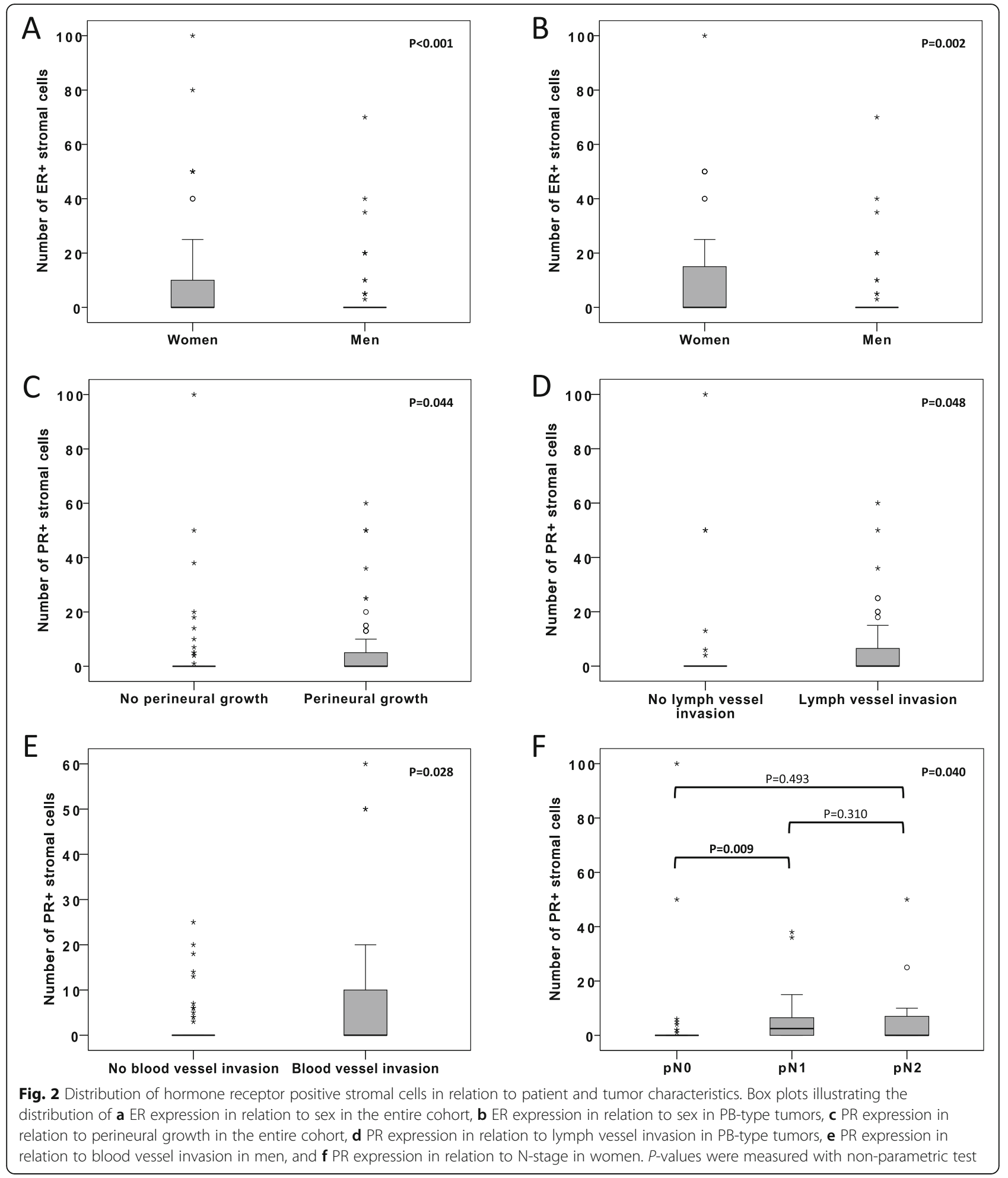

0.18, 95\% CI 0.05-0.60 and HR 0.18, 95\% CI 0.05-0.68, respectively), but not in multivariable analysis. PR+ was not prognostic in subgroup analysis according to tumor origin (data not shown).
Kaplan-Meier analyses of OS and RFS in combined strata of PR expression and KRAS mutation status are shown in Figs. 5 and 6, respectively. In the entire cohort, median OS was 34.3 months for patients with $\mathrm{PR}+$ / 


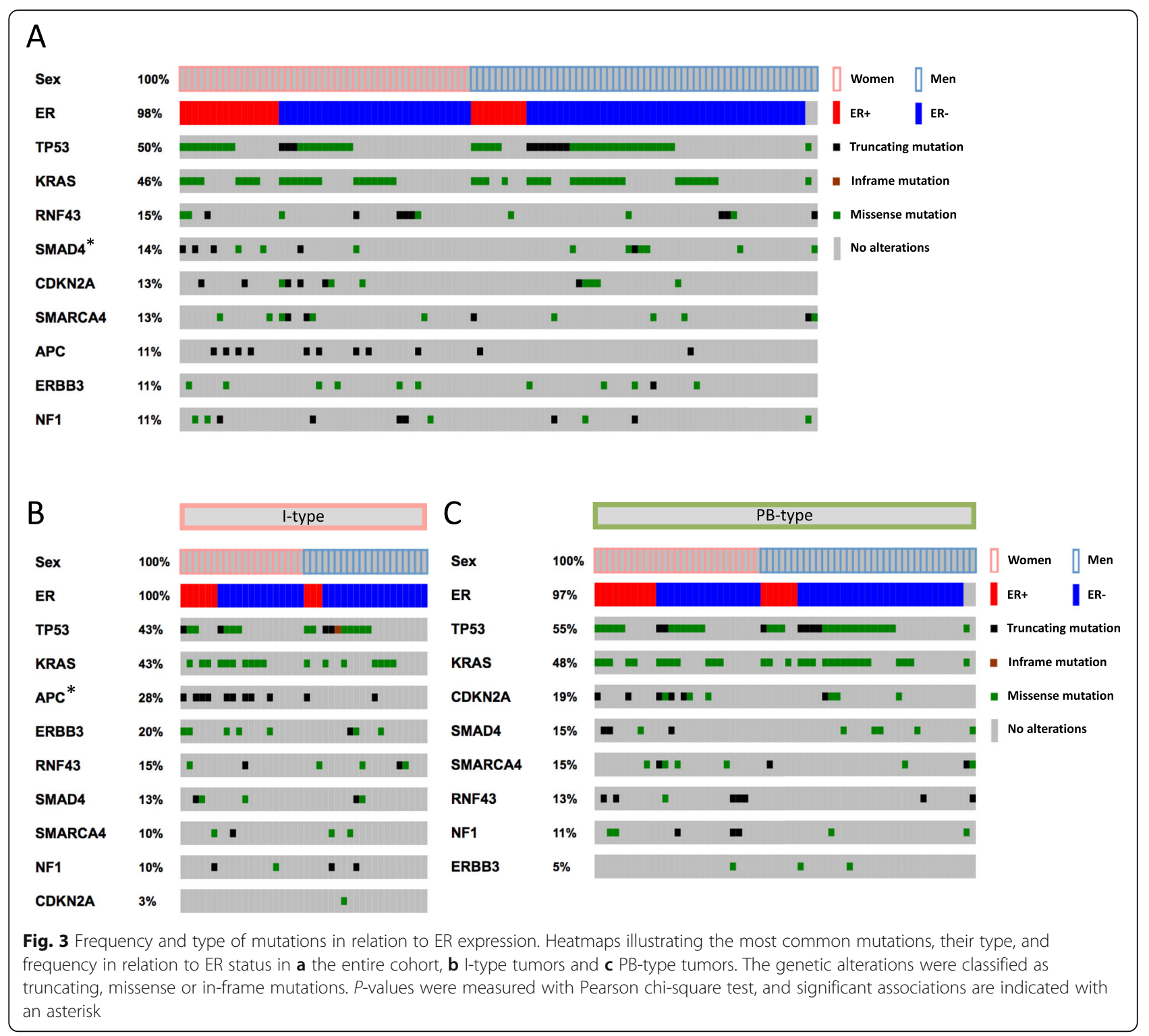

$K R A S$-mutated tumors vs 20.9 months for patients with PR-/KRAS-mutated tumors, and 28.8 months for patients with $\mathrm{PR}+/ K R A S$ wild-type tumors vs 41.8 months for patients with $\mathrm{PR}-/ K R A S$ wild-type tumors. In women, median OS was 60.5 months for patients with $\mathrm{PR}+/ K R A S$-mutated tumors vs 16.6 months for patients with $\mathrm{PR}-/ K R A S$-mutated tumors, and 9.9 months for patients with $\mathrm{PR}+/ K R A S$ wild-type tumors vs 59.0 months for patients with $\mathrm{PR}-/ K R A S$ wild-type tumors.

In the entire cohort, median RFS was 15.0 months for patients with $\mathrm{PR}+/ K R A S$-mutated tumors vs 8.2 months for patients with $\mathrm{PR}-/ K R A S$-mutated tumors, and 19.3 months for patients with $\mathrm{PR}+/ K R A S$ wild-type tumors vs 53.9 months for patients with $\mathrm{PR}-/ K R A S$ wild-type tumors. In women, median RFS was 66.0 months for patients with $\mathrm{PR}+/ K R A S$-mutated tumors vs 6.7 months for patients with $\mathrm{PR}-/ K R A S$-mutated tumors, and 5.1 months for patients with $\mathrm{PR}+/ K R A S$ wild-type tumors vs 79.2 months for patients with $\mathrm{PR}-/ K R A S$-wild-type tumors. Neither ER nor PR expression was prognostic in relation to mutation status of any other of the most frequently mutated genes (data not shown).

\section{Discussion}

This study provides a first description of the prognostic significance of stromal ER and PR expression in pancreatic and other periampullary cancers. Both receptors were expressed mainly in the stromal compartment, in approximately one third of cases but not entirely overlapping. Whilst ER expression was only prognostic in the subgroup of women with pancreatic cancer in unadjusted analysis, stromal PR positivity conferred a 


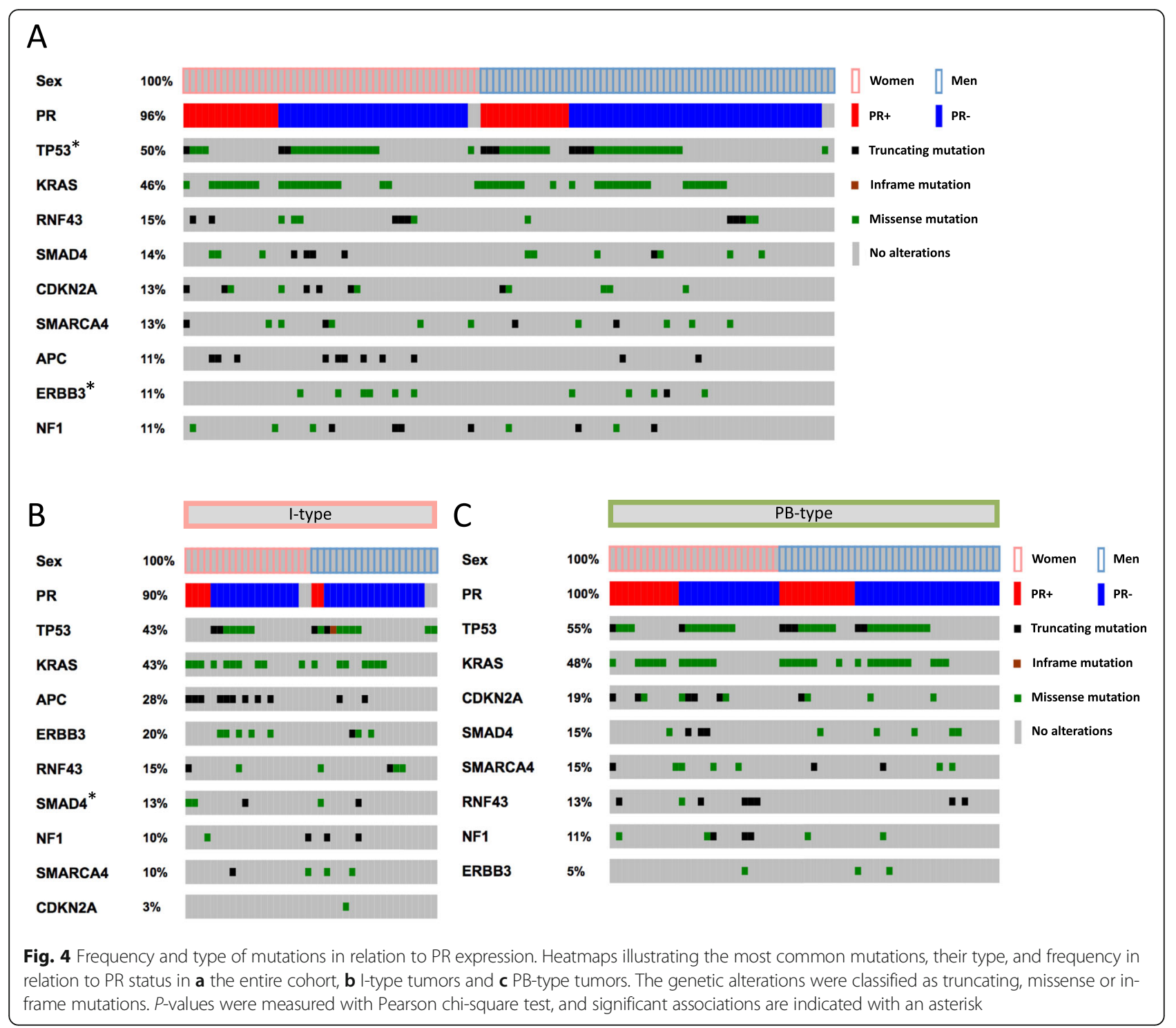

contrasting prognostic value dependent on KRAS mutation status in the entire cohort, and in particular in women. Specifically, KRAS mutated/PR positive tumors signified a prolonged survival, whereas KRAS wild-type/ PR positive tumors signified a reduced survival, and these associations were reflected in both OS and RFS.

The stromal component of ER and PR adjacent to the invasive tumors in this study bears some resemblance to the ovarian-type stroma (OTS) that characterizes pancreatic mucinous cystic neoplasms $(\mathrm{MCN})$, potential premalignant lesions mainly occurring in the tail of the pancreas, and in particular in middle-aged women. The presence of an OTS, together with a lack of communication with the main pancreatic duct, distinguishes these entities from intraductal papillary mucinous neoplasms, another type of premalignant lesion $[44,45]$. The nature of this OTS is debated; while some authors suggest that it constitutes an embryologic remnant of the ovary [46], some speculate that it derives from fetal remnants of periductal mesenchyme exposed to the hormonal environment in perimenopausal women, and that the OTS in turn stimulates the adjacent epithelium to proliferate and give rise to MCN [47]. Loss of PR expression in MCN has been demonstrated to correlate with highgrade dysplasia/carcinoma in-situ or true invasion [48]. Conversely, the frequency of KRAS mutations in MCN increases with the grade of dysplasia and/or invasion [49]. In the present study, no significant associations were found between stromal ER or PR expression and KRAS mutation status, neither overall nor in any subgroup analyses. Moreover, as previously shown, the frequency of KRAS mutations does not differ significantly between I-type and PB-type tumors [40]. The observation of a prognostic interaction of $\mathrm{PR}$ expression and 
Table 1 Unadjusted hazard ratios for death in relation to stromal ER and PR expression, allover and stratified by KRAS mutation status and sex, respectively

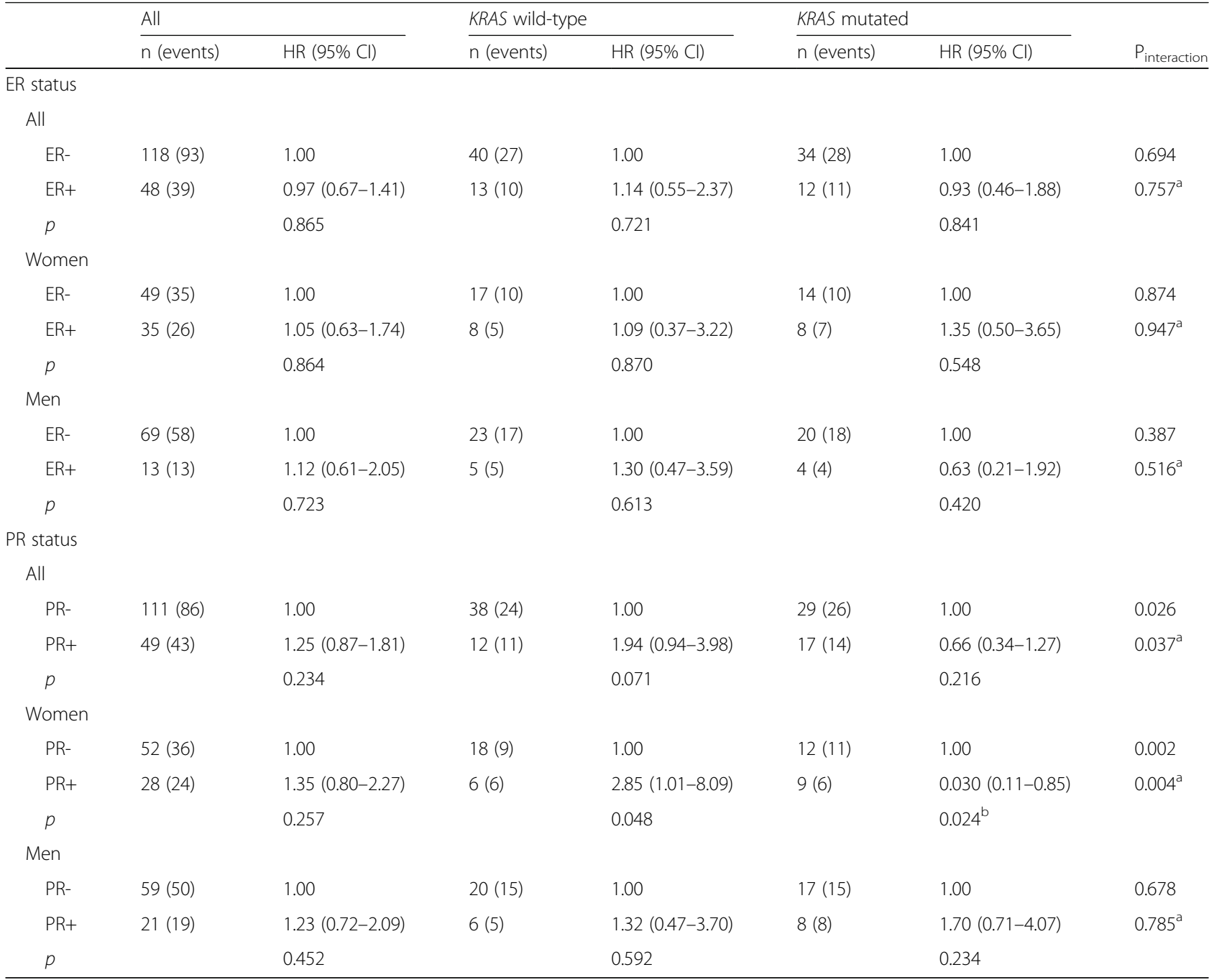

Interaction analysis including adjustment for morphological type (I-type/PB-type)

${ }^{b}$ Hazard ratio adjusted for morphological type $0.28(0.10-0.80)$ in women

KRAS mutation status is however noteworthy, as it suggests that PR-mediated signaling may have tumor suppressing effects in KRAS-mutated tumors and tumorpromoting effects in KRAS wild-type tumors. Speculatively, these effects may be more prominent in the hormonal milieu of the female pancreas, but it must be pointed out that while the findings in this study indicate a potential effect of sex on the prognostic significance of $\mathrm{PR} / K R A S$ mutation status, there was no significant interaction with sex in relation to clinical outcome.

The specific functions of steroid hormone receptors are known to be dependent on the tissue-specific as well as cell-specific context, and, as previously mentioned, the mechanistic basis for their potential action in pancreatic carcinogenesis remains to be explored. Some clues may however be derived from studies on other types of cancer. For instance, the importance of stromal
PR in endometrial cancer has been highlighted in a paper by Janzen et al. [50], wherein the authors demonstrate that the effects of progesterone are mediated via paracrine signaling via PR in the tumor microenvironment. Of particular interest are the findings that concomitant KRAS-activation and PTEN-loss induced progesterone-resistance and loss of stromal PR in endometrial tumors, but that progesterone sensitivity could be reintroduced through exogenous administration of PR into the stroma. The authors conclude that analysis of PTEN and KRAS status in the epithelium of the tumor, and PR expression in the stroma, may be suitable biomarkers for progesterone sensitivity in endometrial cancer [50]. With these data in mind, the finding of a prognostic interaction between KRAS and PR in the present study suggest that further in-depth interrogation into the functional link between KRAS and stromal PR- 
Table 2 Unadjusted hazard ratios for recurrence in relation to stromal ER and PR expression, allover and stratified by KRAS mutation status and sex, respectively

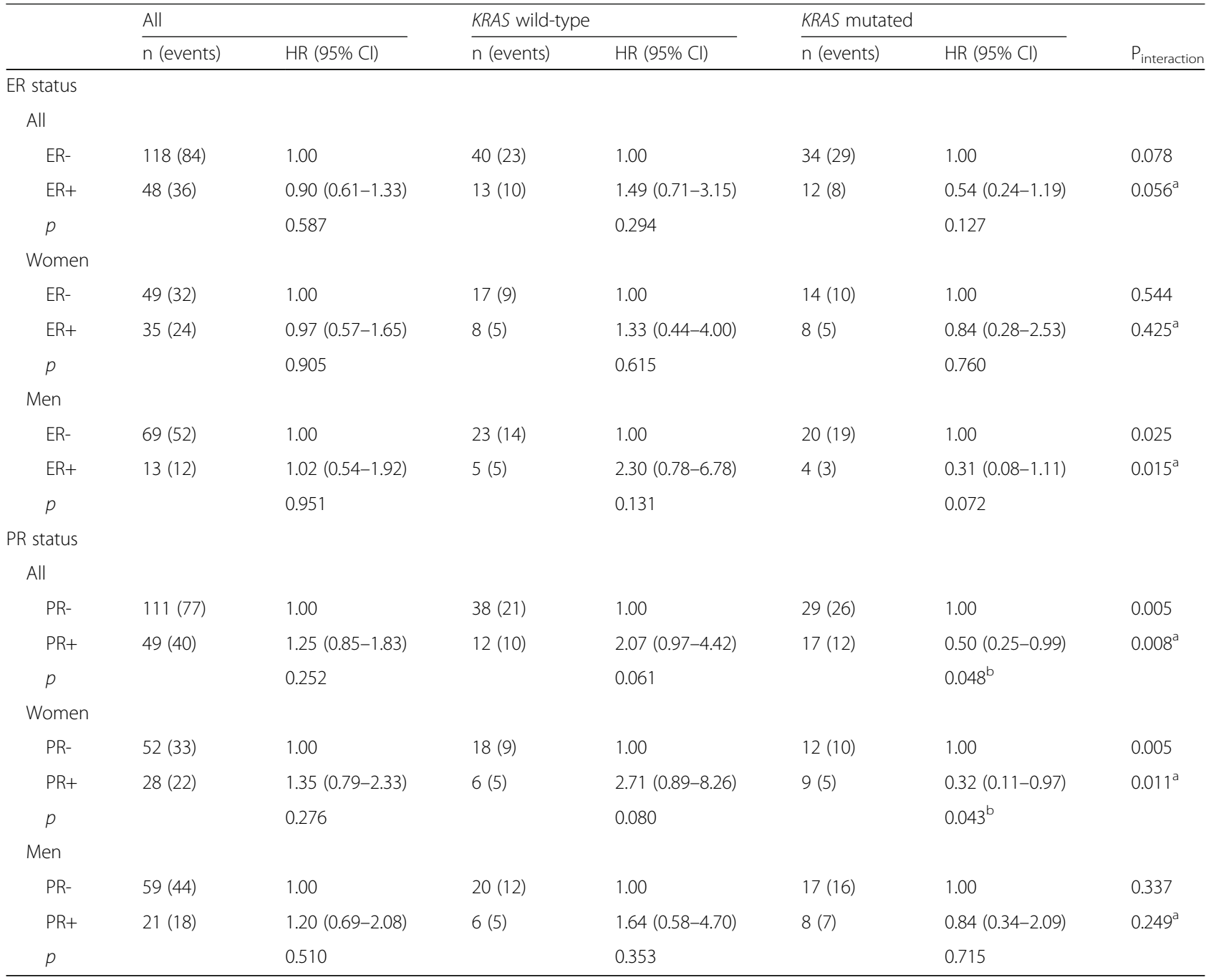

anteraction analysis including adjustment for morphological type (I-type/PB-type)

${ }^{b}$ Hazard ratio adjusted for morphological type $0.46(0.23-0.93)$ in the entire cohort, and $0.32(0.11-0.96)$ in women

signaling in pancreatic and other periampullary cancers may unveil important clues to their pathogenesis and open up for the development of novel treatment strategies.

Herein, well-validated antibodies routinely used in the clinic were applied to detect ER and PR expression, and the expression in tumor cells was found to be negligible. One recent study reported both ER and PR to be weakly expressed in a proportion of 60 pancreatic ductal adenocarcinoma cases [33]. In that study, expression of both ER- $\alpha$ and ER- $\beta$, as well as PR and androgen receptors, was analyzed by an automated approach resulting in an $\mathrm{H}$-score, and while the exact proportion of cases with positive vs negative expression was not denoted, only ER- $\alpha$ expression was found to be a negative prognostic factor, however not in adjusted analysis [33]. Results from two other recent studies suggest that expression of
ER- $\beta$ [51], as well as phosphorylated ER- $\beta$ [52], are negative prognostic factors in pancreatic ductal adenocarcinoma. The issue with a lack of reliable antibodies for detection of ER- $\beta$ has however not yet been resolved [53], and therefore these findings should be interpreted with caution.

A potential limitation to the present study is the use of the TMA technique, which may not accurately reflect the spatial heterogeneity of investigative biomarkers. It must however be pointed out that not even analysis of a whole tissue section from one donor block would help circumvent this limitation. In the TMA used in the present study, each tumor is, with few exceptions, represented by tissue cores derived from different donor blocks.

Another potential limitation is the heterogeneity within periampullary adenocarcinoma. From a clinical 

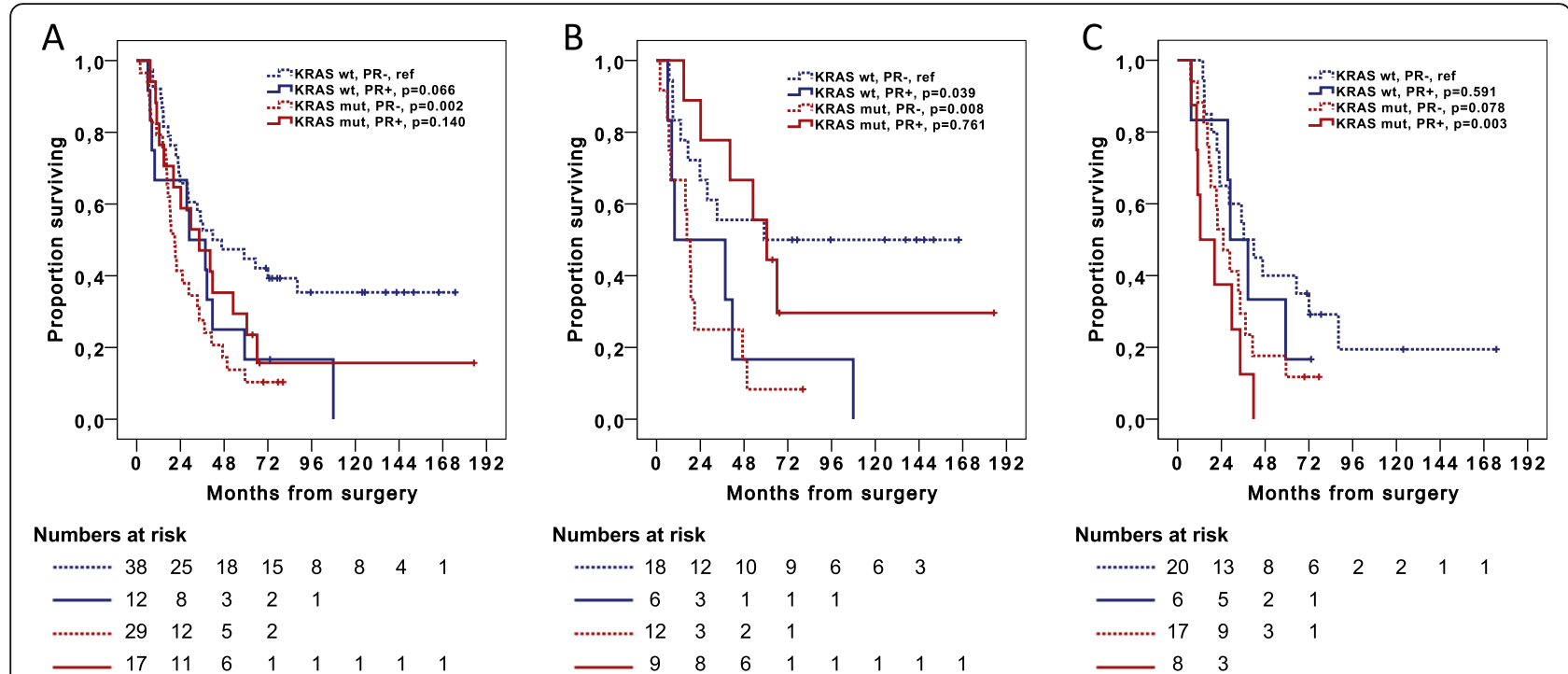

Fig. 5 Associations of KRAS mutation status and PR-expression with overall survival. Kaplan-Meier curves illustrating overall survival in relation to KRAS mutation status and PR status in a all cases, $\mathbf{b}$ women and $\mathbf{c}$ men. P-values were measured with log-rank test

viewpoint however, it is still relevant to consider these tumors as one entity, as anatomic origin cannot always be determined for irresectable tumors, which constitute a vast majority of the cases.

Given the scattered distribution of hormone receptor positive cells from neuroendocrine islets in resected tumor specimens, assessment of ER or PR mRNA levels is not likely to provide reliable prognostic information, but may give some directions. For instance, assessment of ER- $\alpha, E R-\beta$ and PR levels in 176 pancreatic ductal adenocarcinoma cases in The Cancer Genome Atlas (TCGA), as displayed in the Human Protein Atlas/
Pathology Atlas portal (www.proteinatlas.org), reveals an overall low mRNA expression of ER- $\alpha, E R-\beta$ and PR (average FPKM 0.1, 0.2 and 0.4, respectively). Moreover, high levels of PR and ER- $\beta$ are shown to be weakly associated with an improved survival, whereas ER- $\alpha$ is not prognostic. Of note, $>90 \%$ of the TCGA pancreatic cancers are KRAS-mutated. Thus, although the assay is not optimal, the findings regarding PR mRNA expression in the TCGA can be interpreted as being supportive of the herein presented results.

Regarding the associations of other common mutations with stromal ER or PR expression, no clear
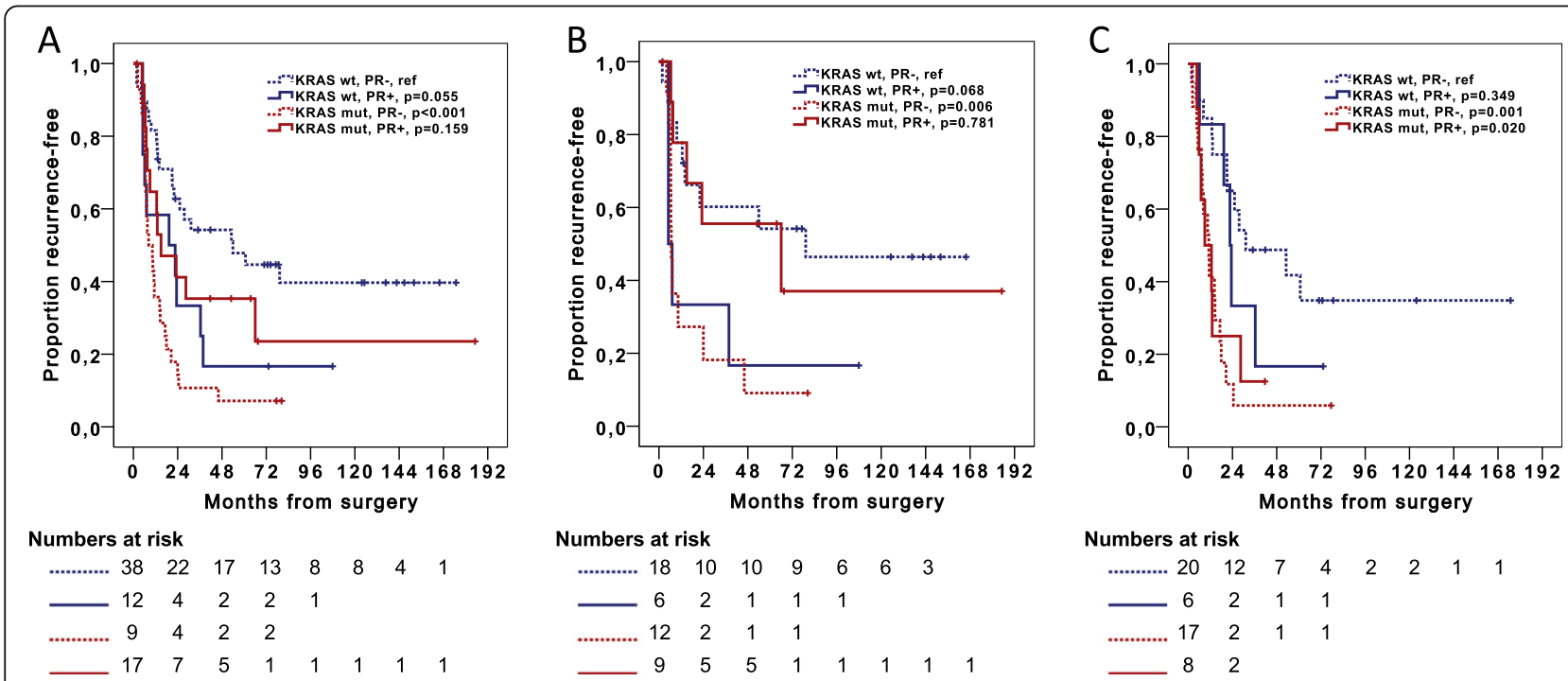

Fig. 6 Associations of KRAS mutation status and PR-expression with recurrence-free survival. Kaplan-Meier curves illustrating recurrence-free survival in relation to KRAS mutation status and PR status in $\mathbf{a}$ all cases, $\mathbf{b}$ women and $\mathbf{c}$ men. P-values were measured with log-rank test 
patterns or associations were found in the present study, and since many subgroups were analyzed, the few significant associations that were found may well be due to type 1 errors. Moreover, although the herein analyzed consecutive cohort of resected periampullary adenocarcinoma is a clinically and histopathologically well-characterized consecutive series of all patients who underwent pancreaticoduodenectomy during an 11-year period, mutation analyses could only be performed in 102 cases from the original cohort of 175 cases, and further stratifying for sex or morphology renders rather small subgroups available for statistical analyses. However, since the outlook for patients afflicted by pancreatic or other periampullary cancers is sinister and treatment options are limited, identification of novel therapies with clinical benefit only in small subgroups would still be a great leap forward.

Although not reporting other than the relationship between stromal ER and PR expression and clinical outcome upon standard treatment in a retrospective, consecutive cohort, the results from this study encourage a revision of some earlier studies that demonstrated beneficial effects of tamoxifen treatment in patients with advanced pancreatic adenocarcinoma [15], in particular in women $[16,17]$. None of these clinical trials included any biomarkers, and the anti-cancer effects of tamoxifen in pancreatic cancer may well be exerted via other mechanisms than blocking of ER-mediated signaling. For instance, tamoxifen has also been shown to confer anti-angiogenic effects [54], partly through inhibition of protein kinase $C[55,56]$, and partly by diminishing the release of vascular endothelial growth factor from thrombocytes [57], and anti-metastatic effects, through prevention of platelet-assisted migration of tumor cells through the endothelium [57]. Nevertheless, a role of female hormone receptors in this context cannot be ruled out.

\section{Conclusion}

The results from this study demonstrate that stromal PR expression, together with KRAS mutation status, provides long-term prognostic information in particular in female patients with pancreatic and other periampullary cancers. Further study into the functional link between KRAS and stromal PR-mediated signaling in these cancers is encouraged, as this may unveil important clues to their pathogenesis and open up for the discovery of novel treatment options. The concept of tamoxifen treatment in patients with advanced disease may also be resurrected, and its therapeutic efficacy tested against biomarkers such as KRAS status and hormone receptor expression.

\section{Supplementary information}

Supplementary information accompanies this paper at https://doi.org/10. 1186/s40364-019-0176-9.

Additional file 1: Data 1. Specification of the targeted gene panel, showing types of mutations and tumor morphology in each case for every 70 genes sequenced.

Additional file 2: Table S1. Intercorrelation between ER and PR expression in the entire cohort and stratified by sex and morphology.

Additional file 3: Table S2. Associations of ER expression status (negative vs positive) with patient and tumor characteristics in the entire cohort, intestinal-type tumors and pancreatobiliary-type tumors, allover and stratified by sex.

Additional file 4: Table S3. Associations of PR expression status (negative vs positive) with patient and tumor characteristics in the entire cohort, intestinal-type tumors and pancreatobiliary-type tumors, allover and stratified by sex.

Additional file 5: Table S4. Associations of ER expression status (negative vs positive) with common mutations in the entire cohort, intestinal-type tumors and pancreatobiliary-type tumors, allover and stratified by sex.

Additional file 6: Table S5. Associations of PR expression status (negative vs positive) with common mutations in the entire cohort, intestinal-type tumors and pancreatobiliary-type tumors, allover and stratified by sex.

\section{Abbreviations}

Cl: Confidence interval; ER: Estrogen receptor-a; FFPE: Formalin-fixed paraffinembedded; HR: Hazard ratio; I-type: Intestinal type; MCN: Mucinous cystic neoplasm; OS: Overall survival; OTS: Ovarian-type stroma; PB-

type: Pancreatobiliary type; PR: Progesterone receptor; RFS: Recurrence-free survival; TCGA: The Cancer Genome Atlas; TMA: Tissue microarray

\section{Acknowledgements}

Not applicable.

\section{Authors' contributions}

GA performed the immunohistochemical and statistical analyses and drafted the manuscript. SL assisted with the statistical analyses and the manuscript drafting. $\mathrm{MH}$ collected clinical data and helped draft the manuscript. BN constructed the TMAs, performed the immunohistochemical stainings, and helped draft the manuscript. JE carried out the histopathological reevaluation, carried out the immunohistochemical analyses, and helped draft the manuscript. $\mathrm{KJ}$ conceived of the study, assisted with the statistical analyses, and helped draft the manuscript. All authors read and approved the final manuscript.

\section{Funding}

This work was supported by grants from The Swedish Research Council (grant number 2015-03598), the Mrs. Berta Kamprad Foundation (grant number FBKS-2018-15), The Swedish Cancer Society (grant number 2016/ 483), Governmental Funding of Clinical Research within the National Health Service (ALF) (grant numbers F 2014/354), Lund University Faculty of Medicine and Skåne University Hospital Funds and Donations.

\section{Availability of data and materials}

Part of the data generated and analyzed during this study are included in this published article. A data sheet of the analyzed genes $(n=70)$, including the NGS data, is provided in Additional file 1: Data 1 as well as in the article by Lundgren et al. [40]. The raw data of hormone receptor expression will be made available upon request. Patient and clinicopathological data of this cohort are not publicly available due to its content of identifiable human data. Requests to access the datasets should be directed to Dr. Gustav Andersson. 


\section{Ethics approval and consent to participate}

The study was approved of by the Ethics committee of Lund University (ref $\mathrm{nr}$ 445/2007), whereby the committee waived no need for consent other than the option to opt out.

\section{Consent for publication}

Not applicable.

\section{Competing interests}

The authors declare that they have no competing interests.

Received: 3 September 2019 Accepted: 25 October 2019 Published online: 19 November 2019

\section{References}

1. Bray F, Ferlay J, Soerjomataram I, Siegel RL, Torre LA, Jemal A. Global cancer statistics 2018: GLOBOCAN estimates of incidence and mortality worldwide for 36 cancers in 185 countries. CA Cancer J Clin. 2018;68(6):394-424.

2. Kimura W, Futakawa N, Zhao B. Neoplastic diseases of the papilla of Vater. J Hepato-Biliary-Pancreat Surg. 2004;11(4):223-31.

3. Carter JT, Grenert JP, Rubenstein L, Stewart L, Way LW. Tumors of the ampulla of vater: histopathologic classification and predictors of survival. J Am Coll Surg. 2008;207(2):210-8.

4. Westgaard A, Tafjord S, Farstad IN, Cvancarova M, Eide TJ, Mathisen O, et al. Pancreatobiliary versus intestinal histologic type of differentiation is an independent prognostic factor in resected periampullary adenocarcinoma. BMC Cancer. 2008;8:170.

5. Bronsert P, Kohler I, Werner M, Makowiec F, Kuesters S, Hoeppner J, et al. Intestinal-type of differentiation predicts favourable overall survival: confirmatory clinicopathological analysis of 198 periampullary adenocarcinomas of pancreatic, biliary, ampullary and duodenal origin. BMC Cancer. 2013:13:428.

6. McGuigan A, Kelly P, Turkington RC, Jones C, Coleman HG, McCain RS. Pancreatic cancer: a review of clinical diagnosis, epidemiology, treatment and outcomes. World J Gastroenterol. 2018;24(43):4846-61.

7. Siegel RL, Miller KD, Jemal A. Cancer statistics, 2016. CA Cancer J Clin. 2016; 66(1):7-30.

8. Gillen S, Schuster T, Meyer Zum Buschenfelde C, Friess H, Kleeff J. Preoperative/neoadjuvant therapy in pancreatic cancer: a systematic review and meta-analysis of response and resection percentages. PLoS Med. 2010; 7(4):e1000267.

9. Conroy T, Desseigne F, Ychou M, Bouche O, Guimbaud R, Becouarn Y, et al. FOLFIRINOX versus gemcitabine for metastatic pancreatic cancer. N Engl J Med. 2011;364(19):1817-25

10. Von Hoff DD, Ervin T, Arena FP, Chiorean EG, Infante J, Moore M, et al. Increased survival in pancreatic cancer with nab-paclitaxel plus gemcitabine. N Engl J Med. 2013:369(18):1691-703.

11. Grant TJ, Hua K, Singh A. Molecular pathogenesis of pancreatic cancer. Prog Mol Biol Transl Sci. 2016:144:241-75.

12. Chau I. Clinical development of PD-1/PD-L1 immunotherapy for gastrointestinal cancers: facts and hopes. Clin Cancer Res. 2017;23(20): 6002-11.

13. Theve NO, Pousette A, Carlstrom K. Adenocarcinoma of the pancreas--a hormone sensitive tumor? A preliminary report on Nolvadex treatment. Clin Oncol. 1983;9(3):193-7.

14. Tonnesen K, Kamp-Jensen M. Antiestrogen therapy in pancreatic carcinoma: a preliminary report. Eur J Surg Oncol. 1986;12(1):69-70.

15. Horimi T, Takasaki M, Toki A, Nishimura W, Morita S. The beneficial effect of tamoxifen therapy in patients with resected adenocarcinoma of the pancreas. Hepatogastroenterology. 1996:43(11):1225-9.

16. Bakkevold KE, Pettersen A, Arnesjo B, Espehaug B. Tamoxifen therapy in unresectable adenocarcinoma of the pancreas and the papilla of Vater. $\mathrm{Br} J$ Surg. 1990;77(7):725-30,

17. Wong A, Chan A. Survival benefit of tamoxifen therapy in adenocarcinoma of pancreas. A case-control study. Cancer. 1993:71(7):2200-3.

18. Kreiger N, Lacroix J, Sloan M. Hormonal factors and pancreatic cancer in women. Ann Epidemiol. 2001:11(8):563-7.

19. Skinner HG, Michaud DS, Colditz GA, Giovannucci EL, Stampfer MJ, Willett WC, et al. Parity, reproductive factors, and the risk of pancreatic cancer in women. Cancer Epidemiol Biomark Prev. 2003;12(5):433-8.
20. Teras LR, Patel AV, Rodriguez C, Thun MJ, Calle EE. Parity, other reproductive factors, and risk of pancreatic cancer mortality in a large cohort of U.S. women (United States). Cancer Causes Control. 2005;16(9):1035-40.

21. Lin Y, Kikuchi S, Tamakoshi A, Kawamura T, Inaba Y, Kurosawa M, et al. Association of menstrual and reproductive factors with pancreatic cancer risk in women: findings of the Japan collaborative cohort study for evaluation of cancer risk. J Gastroenterol. 2006;41(9):878-83.

22. Prizment $A E$, Anderson KE, Hong CP, Folsom AR. Pancreatic cancer incidence in relation to female reproductive factors: lowa women's health study. JOP. 2007;8(1):16-27.

23. Zhang Y, Coogan PF, Palmer JR, Strom BL, Rosenberg L. A case-control study of reproductive factors, female hormone use, and risk of pancreatic cancer. Cancer Causes Control. 2010;21(3):473-8.

24. Lucenteforte E, Zucchetto A, Bosetti C, Talamini R, Negri E, Serraino D, et al. Reproductive and hormonal factors and pancreatic cancer risk in women. Pancreas. 2011;40(3):460-3

25. Lee E, Horn-Ross PL, Rull RP, Neuhausen SL, Anton-Culver H, Ursin G, et al. Reproductive factors, exogenous hormones, and pancreatic cancer risk in the CTS. Am J Epidemiol. 2013;178(9):1403-13.

26. Andersson G, Borgquist S, Jirstrom K. Hormonal factors and pancreatic cancer risk in women: the Malmo diet and cancer study. Int J Cancer. 2018; 143(1):52-62.

27. Bueno de Mesquita HB, Maisonneuve P, Moerman CJ, Walker AM Anthropometric and reproductive variables and exocrine carcinoma of the pancreas: a population-based case-control study in The Netherlands. Int J Cancer. 1992;52(1):24-9.

28. Fernandez E, La Vecchia C, D'Avanzo B, Negri E. Menstrual and reproductive factors and pancreatic cancer risk in women. Int J Cancer. 1995:62(1):11-4.

29. Ji BT, Hatch MC, Chow WH, MCLaughlin JK, Dai Q, Howe GR, et al. Anthropometric and reproductive factors and the risk of pancreatic cancer: a case-control study in Shanghai, China. Int J Cancer. 1996;66(4):432-7.

30. Karlson BM, Wuu J, Hsieh CC, Lambe M, Ekbom A. Parity and the risk of pancreatic cancer: a nested case-control study. Int J Cancer. 1998;77(2):224-7.

31. Duell EJ, Holly EA. Reproductive and menstrual risk factors for pancreatic cancer: a population-based study of San Francisco Bay Area women. Am J Epidemiol. 2005;161(8):741-7.

32. Greenway B, lqbal MJ, Johnson PJ, Williams R. Oestrogen receptor proteins in malignant and fetal pancreas. Br Med J (Clin Res Ed). 1981;283(6294):751-3.

33. Georgiadou D, Sergentanis TN, Sakellariou S, Vlachodimitropoulos D, Psaltopoulou T, Lazaris AC, et al. Prognostic role of sex steroid receptors in pancreatic adenocarcinoma. Pathol Res Pract. 2016;212(1):38-43.

34. Elebro J, Jirstrom K. Use of a standardized diagnostic approach improves the prognostic information of histopathologic factors in pancreatic and periampullary adenocarcinoma. Diagn Pathol. 2014;9:80.

35. Elebro J, Heby M, Gaber A, Nodin B, Jonsson L, Fristedt R, et al. Prognostic and treatment predictive significance of SATB1 and SATB2 expression in pancreatic and periampullary adenocarcinoma. J Transl Med. 2014;12:289.

36. Fristedt R, Elebro J, Gaber A, Jonsson L, Heby M, Yudina Y, et al. Reduced expression of the polymeric immunoglobulin receptor in pancreatic and periampullary adenocarcinoma signifies tumour progression and poor prognosis. PLoS One. 2014;9(11):e112728.

37. Heby M, Elebro J, Nodin B, Jirstrom K, Eberhard J. Prognostic and predictive significance of podocalyxin-like protein expression in pancreatic and periampullary adenocarcinoma. BMC Clin Pathol. 2015;15:10.

38. Elebro J, Ben Dror L, Heby M, Nodin B, Jirstrom K, Eberhard J. Prognostic effect of hENT1, dCK and HuR expression by morphological type in periampullary adenocarcinoma, including pancreatic cancer. Acta Oncol. 2016;55(3):286-96.

39. Heby M, Lundgren S, Nodin B, Elebro J, Eberhard J, Jirstrom K. Relationship between mismatch repair immunophenotype and long-term survival in patients with resected periampullary adenocarcinoma. J Transl Med. 2018;16(1):66.

40. Lundgren S, Olsson Hau S, Elebro J, Heby M, Karnevi E, Nodin B, et al. Mutational landscape in resected periampullary adenocarcinoma: relationship with morphology and clinical outcome. JCO Precision Oncol. 2019:3:1-8.

41. Cerami E, Gao J, Dogrusoz U, Gross BE, Sumer SO, Aksoy BA, et al. The cBio cancer genomics portal: an open platform for exploring multidimensional cancer genomics data. Cancer Discov. 2012;2(5):401-4.

42. Gao J, Aksoy BA, Dogrusoz U, Dresdner G, Gross B, Sumer SO, et al. Integrative analysis of complex cancer genomics and clinical profiles using the cBioPortal. Sci Signal. 2013;6(269):pl1. 
43. Bender R, Lange S. Adjusting for multiple testing--when and how? J Clin Epidemiol. 2001;54(4):343-9.

44. Crippa S, Salvia R, Warshaw AL, Dominguez I, Bassi C, Falconi M, et al. Mucinous cystic neoplasm of the pancreas is not an aggressive entity: lessons from 163 resected patients. Ann Surg. 2008;247(4):571-9.

45. Ishida K, Sasano H, Moriya T, Takahashi Y, Sugimoto R, Mue Y, et al. Immunohistochemical analysis of steroidogenic enzymes in ovarian-type stroma of pancreatic mucinous cystic neoplasms: comparative study of subepithelial stromal cells in intraductal papillary mucinous neoplasms of the pancreas. Pathol Int. 2016;66(5):281-7.

46. Izumo A, Yamaguchi K, Equchi T, Nishiyama K, Yamamoto H, Yonemasu $\mathrm{H}$, et al. Mucinous cystic tumor of the pancreas: immunohistochemical assessment of "ovarian-type stroma". Oncol Rep. 2003;10(3):515-25.

47. Reid MD, Saka B, Balci S, Goldblum AS, Adsay NV. Molecular genetics of pancreatic neoplasms and their morphologic correlates: an update on recent advances and potential diagnostic applications. Am J Clin Pathol. 2014;141(2):168-80.

48. Jang KT, Park SM, Basturk O, Bagci P, Bandyopadhyay S, Stelow EB, et al. Clinicopathologic characteristics of 29 invasive carcinomas arising in 178 pancreatic mucinous cystic neoplasms with ovarian-type stroma: implications for management and prognosis. Am J Surg Pathol. 2015;39(2): 179-87.

49. Jimenez RE, Warshaw AL, Z'Graggen K, Hartwig W, Taylor DZ, Compton CC, et al. Sequential accumulation of K-ras mutations and p53 overexpression in the progression of pancreatic mucinous cystic neoplasms to malignancy. Ann Surg. 1999:230(4):501-9 discussion 9-11.

50. Janzen DM, Rosales MA, Paik DY, Lee DS, Smith DA, Witte ON, et al. Progesterone receptor signaling in the microenvironment of endometrial cancer influences its response to hormonal therapy. Cancer Res. 2013;73(15): 4697-710.

51. Seeliger H, Pozios I, Assmann G, Zhao Y, Muller MH, Knosel T, et al. Expression of estrogen receptor beta correlates with adverse prognosis in resected pancreatic adenocarcinoma. BMC Cancer. 2018;18(1):1049.

52. Pozios I, Knosel T, Zhao Y, Assmann G, Pozios I, Muller MH, et al. Expression of phosphorylated estrogen receptor beta is an independent negative prognostic factor for pancreatic ductal adenocarcinoma. J Cancer Res Clin Oncol. 2018:144(10):1887-97.

53. Andersson S, Sundberg M, Pristovsek N, Ibrahim A, Jonsson P, Katona B, et al. Insufficient antibody validation challenges oestrogen receptor beta research. Nat Commun. 2017;8:15840.

54. Gagliardi A, Collins DC. Inhibition of angiogenesis by antiestrogens. Cancer Res. 1993;53(3):533-5.

55. O'Brian CA, Liskamp RM, Solomon DH, Weinstein IB. Inhibition of protein kinase C by tamoxifen. Cancer Res. 1985;45(6):2462-5.

56. Su HD, Mazzei GJ, Vogler WR, Kuo JF. Effect of tamoxifen, a nonsteroidal antiestrogen, on phospholipid/calcium-dependent protein kinase and phosphorylation of its endogenous substrate proteins from the rat brain and ovary. Biochem Pharmacol. 1985:34(20):3649-53.

57. Johnson KE, Forward JA, Tippy MD, Ceglowski JR, El-Husayni S, Kulenthirarajan $\mathrm{R}$, et al. Tamoxifen directly inhibits platelet angiogenic potential and platelet-mediated metastasis. Arterioscler Thromb Vasc Biol. 2017;37(4):664-74

\section{Publisher's Note}

Springer Nature remains neutral with regard to jurisdictional claims in published maps and institutional affiliations.

\section{Ready to submit your research? Choose BMC and benefit from:}

- fast, convenient online submission

- thorough peer review by experienced researchers in your field

- rapid publication on acceptance

- support for research data, including large and complex data types

- gold Open Access which fosters wider collaboration and increased citations

- maximum visibility for your research: over $100 \mathrm{M}$ website views per year

At $\mathrm{BMC}$, research is always in progress.

Learn more biomedcentral.com/submissions 